\title{
EXISTENCE OF REGULAR LAGRANGE MULTIPLIERS FOR A NONLINEAR ELLIPTIC OPTIMAL CONTROL PROBLEM WITH POINTWISE CONTROL-STATE CONSTRAINTS
}

\author{
A. RÖSCH $*$ AND F. TRÖLTZSCH ${ }^{\dagger}$
}

\begin{abstract}
A class of optimal control problems for semilinear elliptic equations with mixed control-state constraints is considered. The existence of bounded and measurable Lagrange multipliers is proven. As a particular application, the Lavrentiev type regularization of pointwise state constraints is discussed. Here, the existence of associated regular multipliers is shown, too.
\end{abstract}

Key words. Nonlinear programming, function space, optimal control, Lagrange multiplier, semilinear elliptic equation, mixed control-state constraint, pointwise state constraint, Lavrentiev type regularization.

AMS subject classifications. 49K20, 49N10, 49N15, 90C45

1. Introduction. In this paper, we consider the optimal control problem

$$
\min J(y, u):=\int_{\Omega} \psi(x, y(x), u(x)) d x+\int_{\Gamma} \varphi(x, y(x)) d s(x)
$$

subject to the semilinear boundary value problem

$$
\begin{aligned}
(A y)(x)+d(x, y(x)) & =\beta(x) u(x) & & \text { in } \Omega \\
\partial_{\nu} y(x)+b(x, y(x)) & =0 & & \text { on } \Gamma
\end{aligned}
$$

and to the mixed control-state constraints

$$
\begin{array}{ll}
0 \leq u(x) \leq c(x)+\gamma(x) y(x) & \text { a.e. in } \Omega \\
u(x) \leq e(x) & \text { a.e. in } \Omega .
\end{array}
$$

We will investigate also another type of constraints that might be useful for applications. In this setting, $A$ is a uniformly elliptic differential operator and $\Omega$ is a bounded domain of $\mathbb{R}^{N}, N \geq 2$, with boundary $\Gamma$ and outward unit normal $\nu$. Precise assumptions on and definitions of the quantities introduced above are formulated at the end of this section.

The investigation of optimal control problems of this type is interesting for different reasons: First of all, they exhibit nice theoretical properties. As we shall prove in this paper, the Lagrange multipliers associated with optimal solutions can assumed to be quite regular functions, while they are measures for pure state constraints. This higher regularity allows for a better numerical analysis. For instance, second-order sufficient optimality conditions can be discussed in a fairly complete way, [15], [14]. For pointwise state constraints, the associated theory is still partially open, cf. Casas et al. [7], since it works only for small dimension of $\Omega$. Moreover, we mention the application of mixed constraints in the numerical analysis that is briefly addressed in Section 7 .

Constraints of this type are also interesting for problems, where the difference between control and state has to be bounded (problems of bottleneck type). An application to optimal heating has been given in [19].

*Johann Radon Institute for Computational and Applied Mathematics (RICAM), Austrian Academy of Sciences, Altenbergerstraße 69, A-4040 Linz, Austria, arnd.roesch@oeaw.ac.at

${ }^{\dagger}$ Technische Universität Berlin, Fakultät II - Mathematik und Naturwissenschaften, Str. des 17. Juni 136, D-10623 Berlin, Germany, troeltzsch@math.tu-berlin.de 
Our main issue is the existence of bounded and measurable Lagrange multipliers associated with the constraints (1.3). We shall consider the control $u$ in $L^{\infty}(\Omega)$ - this is the natural space being obtained from the constraints. Therefore, the multipliers should be expected in the dual space $L^{\infty}(\Omega)^{*}$, a space of Radon measures.

However, it is known from similar problems for linear and nonlinear parabolic problems that the Lagrange multipliers can be expected to be more regular under natural assumptions [3], [4], [5], [18]. In fact, they can be constructed as functions of $L^{2}(\Omega)$ or even in $L^{\infty}(\Omega)$. Recently, the same result has been obtained for linearquadratic elliptic problems by convex duality theory, [17]. The elliptic case is more difficult than the parabolic one due to the appearance of eigenvalues.

In principle, two ways are known to prove regularity. In [4], [5], the problems are linearized at the optimal solution and the Lagrange multipliers are obtained by the associated linear dual problem. Another technique has been suggested later in [3]: An application of a Kuhn-Tucker theorem in Banach spaces delivers existence of Lagrange multipliers in $L^{\infty}(\Omega)^{*}$. In a second step, these multipliers are shown to be bounded functions. This step is fairly technical.

Here, we follow again the idea of linearization and application of duality theory. We think that this approach is more elementary, although not simple as well. Moreover, linearization and working with different norms and spaces seems to be interesting on its own. Therefore, we devote a section to this issue. Later, we shall embed the problem (1.1)-(1.3) into a more general class of problems in function spaces and prove the regularity of Lagrange multipliers for this general class. The result for (1.1)-(1.3) is obtained as a conclusion.

Assumptions. $\Omega \subset \mathbb{R}^{N}, N \geq 2$ is a bounded Lipschitz domain. The functions $\psi=\psi(x, y, u): \Omega \times \mathbb{R}^{2} \rightarrow \mathbb{R}, d=d(x, y): \Omega \times \mathbb{R} \rightarrow \mathbb{R}$, and $\varphi, b: \Gamma \times \mathbb{R} \rightarrow \mathbb{R}$ are measurable with respect to $x$ for all fixed pairs $(y, u)$ and differentiable with respect to $y$ and $u$ for all fixed $x$. They satisfy the following conditions on boundedness and Lipschitz continuity: There is a constant $K>0$ and, for all $M>0$, a constant $L(M)>0$ exists such that

$$
\begin{aligned}
& \left|\psi(x, 0,0)+\frac{\partial \psi}{\partial y}(x, 0,0)+\frac{\partial \psi}{\partial u}(x, 0,0)\right| \leq K \\
& \left|\psi\left(x, y_{1}, u_{1}\right)-\psi\left(x, y_{2}, u_{2}\right)\right|+\left|\frac{\partial \psi}{\partial y}\left(x, y_{1}, u_{1}\right)-\frac{\partial \psi}{\partial y}\left(x, y_{2}, u_{2}\right)\right| \\
& +\left|\frac{\partial \psi}{\partial u}\left(x, y_{1}, u_{1}\right)-\frac{\partial \psi}{\partial u}\left(x, y_{2}, u_{2}\right)\right| \leq L(M)\left\{\left|y_{1}-y_{2}\right|+\left|u_{1}-u_{2}\right|\right\}
\end{aligned}
$$

for all $u_{i}, y_{i}$ with $\left|u_{i}\right|+\left|y_{i}\right| \leq M, i=1,2$, and for a.a. $x \in \Omega$. The functions $d, \varphi, b$ satisfy the same assumptions with respect to $x \in \Omega$ or $x \in \Gamma$, respectively.

The functions $\beta, \gamma, c, e: \Omega \rightarrow \mathbb{R}$ are bounded, measurable and nonnegative. To avoid a trivial problem, we assume that $\beta \not \equiv 0 . A$ is a uniformly elliptic differential operator defined by

$$
(A y)(x)=-\sum_{i, j=1}^{N} \frac{\partial}{\partial x_{i}}\left(a_{i j}(x) \frac{\partial}{\partial x_{j}} y(x)\right)+c_{0}(x) y(x)
$$

with functions $a_{i j}$ that belong to $C^{0,1}(\bar{\Omega})$, satisfy the condition $a_{i j}(x)=a_{j i}(x)$ and the condition of uniform ellipticity

$$
\sum_{i, j=1}^{N} a_{i j}(x) \xi_{i} \xi_{j} \geq \delta_{0}|\xi|^{2} \quad \text { a.e. on } \Omega
$$


with some $\delta_{0}>0$ for all $\xi \in \mathbb{R}^{N}$. We assume $c_{0}(x) \geq 0$ a.e. on $\Omega$ and $\left\|c_{0}\right\|_{L^{2}(\Omega)}>0$. It holds $\partial d / \partial y(x, y) \geq 0$ and $\partial b / \partial y(x, y) \geq 0$ for all $y \in \mathbb{R}$ and almost all $x \in \Omega$ and $\Gamma$, respectively.

2. Lagrange multipliers and linearization. In (1.1)-(1.3), the state $y$ is associated with $u$ by a differentiable mapping $G, y=G(u)$, so that $y$ can be eliminated from the problem. Then $J(y, u)=J(G(u), u)=f(u)$, and the state $y$ can also be eliminated in (1.3). Details are worked out in the next section. Finally, we arrive at a differentiable mathematical programming problem in Banach spaces that admits the following form:

$$
\min f(u), \quad g(u) \leq_{K} 0, \quad u \in C .
$$

Here, $f: U \rightarrow \mathbb{R}, g: U \rightarrow Z$ are continuously Fréchet differentiable mappings defined in real Banach spaces $U, Z$. Moreover, $C \subset U$ is a convex set and $K \subset Z$ is a convex closed cone that defines a partial ordering $\leq_{K}$ in $Z$ by $z \leq_{K} 0 \Leftrightarrow-z \in K$. In this sense, $K$ can be considered as the nonnegative cone of $Z$, although the concrete meaning can be quite different.

Assume that $\bar{u}$ is a local solution of (2.1), i.e., for some $r>0$ it holds $f(\bar{u}) \leq f(u)$ for all $u \in U$ with $\|u-\bar{u}\|_{U} \leq r, g(u) \leq_{K} 0$ and $u \in C$. A Lagrange multiplier is an element of the dual cone $K_{+}$of $K$,

$$
K_{+}=\left\{\mu \in Z^{*}: \mu(z) \geq 0 \quad \forall z \in K\right\},
$$

where $Z^{*}$ is the dual space of $Z$, the Banach space of all linear and continuous functionals on $Z$.

Definition 2.1. A Lagrange multiplier associated with $\bar{u}$ is an element $\mu \in K_{+}$ such that the variational inequality

$$
f^{\prime}(\bar{u})(u-\bar{u})+\mu\left(g^{\prime}(\bar{u})(u-\bar{u})\right) \geq 0 \quad \forall u \in C
$$

and the complementary slackness condition

$$
\mu(g(\bar{u}))=0
$$

hold true.

Remark. (2.2) might be written as

$$
\left\langle f^{\prime}(\bar{u})+g^{\prime}(\bar{u})^{*} \mu, u-\bar{u}\right\rangle \geq 0 \quad \forall u \in C
$$

with the adjoint operator $g^{\prime}(\bar{u})^{*}$ and the pairing $\langle\cdot, \cdot\rangle$ between $Z^{*}$ and $Z$. We avoid $g^{\prime}(\bar{u})^{*}$ since it might be fairly complicated to find its concrete form in applications. Moreover, we aim at finding $\mu$ in a more "regular" subspace of $Z^{*}$.

In our applications, $Z$ is of type $L^{\infty}(\Omega)$ and $K$ is the associated nonnegative cone. It has a nonempty interior int $K$. Therefore, we are justified to require the following constraint qualification (regularity of $\bar{u}$ ):

There is a $u_{0} \in C$ such that

$$
-\left[g(\bar{u})+g^{\prime}(\bar{u})\left(u_{0}-\bar{u}\right)\right] \in \operatorname{int} K .
$$

Under this assumption, a Lagrange multiplier $\mu \in Z^{*}$ exists, since (2.4) is equivalent to the well-known regularity condition of Zowe and Kurcyusz [23], see [13] and [20]. 
The condition of Zowe and Kurcyusz is sufficient for the existence of $\mu \in Z^{*}$, [23]. However, we shall apply another conclusion of (2.4) that was used in [23] as an auxiliary result: (2.4) permits to view $\bar{u}$ as the solution of the linearized problem.

Definition 2.2. (Linearizing cone). Let $\bar{z} \in K$ and $\bar{u} \in C$.

$$
\begin{aligned}
& C(\bar{u})=\{v \in U: v=\lambda(u-\bar{u}), \lambda \geq 0, u \in C\} \\
& K(\bar{z})=\{w \in Z: w=\lambda(z-\bar{z}), \lambda \geq 0, z \in K\} .
\end{aligned}
$$

The set

$$
L(\bar{u})=\left\{v \in U: v \in C(\bar{u}), g^{\prime}(\bar{u}) v \in-K(-g(\bar{u}))\right\}
$$

is said to be the linearizing cone of the feasible set at $\bar{u}$.

LEMMA 2.3. Under the regularity assumption (2.4), it holds

$$
f^{\prime}(\bar{u}) v \geq 0 \quad \forall v \in L(\bar{u}) .
$$

For this result of [23] we refer to [20], Theorem 1.2.1 and condition (2.2), [20]. The reason for $(2.5)$ is that $(2.4)$ ensures $L(\bar{u}) \subset T(\bar{u})$, where $T(\bar{u})$ is the so-called tangent cone.

We re-write (2.5) in a more convenient form. Obviously,

$$
L(\bar{u})=\left\{v \in U: v=\alpha(u-\bar{u}), g^{\prime}(\bar{u}) v+\beta g(\bar{u}) \leq_{K} 0, u \in C, \alpha \geq 0, \beta \geq 0\right\} .
$$

Taking $\alpha=\beta=1$, we obtain from (2.5)

$$
f^{\prime}(\bar{u})(u-\bar{u}) \geq 0
$$

for all $u \in C$ with

$$
g(\bar{u})+g^{\prime}(\bar{u})(u-\bar{u}) \leq_{K} 0 .
$$

THEOREM 2.4. If $\bar{u}$ is a local solution of the problem (2.1) that satisfies the constraint qualification (2.4), then $\bar{u}$ is a (global) solution of the linearized programming problem

$$
\min f^{\prime}(\bar{u}) u, \quad g(\bar{u})+g^{\prime}(\bar{u})(u-\bar{u}) \leq_{K} 0, \quad u \in C .
$$

Proof. This follows from re-writing (2.6) in the form $f^{\prime}(\bar{u}) u \geq f^{\prime}(\bar{u}) \bar{u}$, together with (2.7).

THEOREM 2.5. Let the assumptions of Theorem 2.4 be satisfied. Then a Lagrange multiplier $\mu \in K_{+}$associated with $\bar{u}$ exists. It is also a Lagrange multiplier for $\bar{u}$, considered as the solution of (2.8). Conversely, let $\bar{u}$ solve (2.8) and let $\mu$ be any Lagrange multiplier for $\bar{u}$ in (2.8). Then $\bar{u}$ and $\mu$ together satisfy the first-order necessary conditions for problem (2.1).

Proof. Define $\tilde{f}(u)=f^{\prime}(\bar{u}) u, \tilde{g}(u)=g(\bar{u})+g^{\prime}(\bar{u})(u-\bar{u})$. Then we have for all $u$

$$
\tilde{f}^{\prime}(u) \equiv f^{\prime}(\bar{u}), \quad \tilde{g}^{\prime}(u) \equiv g^{\prime}(\bar{u}), \quad \tilde{g}(\bar{u})=g(\bar{u}) .
$$

The first statement of the theorem follows from [23], since the regularity condition (2.4) implies the one of Zowe and Kurcyusz. The other direction is shown as follows: Let $\mu$ be any Lagrange multiplier associated with $\bar{u}$ as a solution of 
(2.8). By the associated complementarity condition and the relations above, we get $\mu(g(\bar{u}))=\mu(\tilde{g}(\bar{u}))=0$. Moreover,

$$
f^{\prime}(\bar{u})(u-\bar{u})+\mu\left(g^{\prime}(\bar{u})(u-\bar{u})\right)=\tilde{f}^{\prime}(\bar{u})(u-\bar{u})+\mu\left(\tilde{g}^{\prime}(\bar{u})(u-\bar{u})\right) \geq 0 \quad \forall u \in C .
$$

Therefore, (2.2) and (2.3) are satisfied so that $\mu$ is a Lagrange multiplier for (2.1).

In view of this, we may concentrate on the linearized problem (2.8) to find a Lagrange multiplier. Why this can be helpful? There are two reasons for: (2.8) can be considered in a space with coarser topology, where $f$ and $g$ are not differentiable. Moreover, we shall be able to use cones of nonnegative functions with empty interior such as $\left(L^{2}(\Omega)\right)_{+}$.

3. Transformation and linearization of the control problem. In this section, we convert the elliptic optimal control problem (1.1)-(1.3) to a mathematical programming problem and consider its linearization, together with an associated dual problem.

THEOREM 3.1. Under the general assumptions, for all $u \in L^{p}(\Omega), p>N / 2$, the equation (1.2) has a unique solution $y=y(u) \in H^{1}(\Omega) \cap C(\bar{\Omega})=: Y$. The associated control-to-state mapping $G: u \mapsto y$ is continuously Fréchet-differentiable. Its derivative $G^{\prime}(\bar{u})$ at $\bar{u} \in L^{p}(\Omega)$ is given by $G^{\prime}(\bar{u}) u=v$, where $v$ solves the linearized equation

$$
\begin{aligned}
(A v)(x)+d_{y}(x, \bar{y}(x)) v(x) & =\beta(x) u(x) & \text { in } \Omega \\
\partial_{\nu} v(x)+b_{y}(x, \bar{y}(x)) v(x) & =0 & \text { on } \Gamma
\end{aligned}
$$

and $\bar{y}=G(\bar{u})$.

This result is meanwhile standard. Existence of $y \in L^{\infty}(\Omega)$ follows for Lipschitz domains by a truncation technique and the Stampacchia method as in Casas [6] or Alibert and Raymond [1]. In [6], the continuity of $y$ was still shown for $C^{1,1}$-domains. In [1], continuity has been deduced from a result by Murthy and Stampacchia [12] that holds in Lipschitz domains. The reader might also consult the monography [21], where these ideas are presented quite detailed in the Sections 4.1.3 and 7.1.2.

In what follows, we consider $G$ and $G^{\prime}(\bar{u})$ as operators with range in $C(\bar{\Omega})$ although their range is actually contained also in $H^{1}(\Omega)$. Inserting $y=G(u)$ in $J$, the elliptic problem (1.1)-(1.3) is transformed to

$$
\min f(u):=J(G(u), u)
$$

subject to

$$
\begin{aligned}
0 \leq u(x) \leq c(x)+\gamma(x)(G(u))(x) \\
u(x) \leq e(x)
\end{aligned}
$$

for almost all $x \in \Omega$. If we consider $u$ as a function of $L^{p}(\Omega)$, then $0 \leq u(x) \leq e(x)$ and the assumption $e \in L^{\infty}(\Omega)$ guarantee $u \in L^{\infty}(\Omega)$. Therefore, from now on, we fix $U=L^{\infty}(\Omega), Z=\left(L^{\infty}(\Omega)\right)^{2}, K=\left(L^{\infty}(\Omega)_{+}\right)^{2}$ and we define $g: U \rightarrow Z$ by

$$
(g(u))(x)=\left(\begin{array}{l}
u(x)-c(x)-\gamma(x)(G(u))(x) \\
u(x)-e(x)
\end{array}\right) .
$$

Moreover, we define $C=L^{\infty}(\Omega)_{+}$. Then (1.1)-(1.3) is equivalent to

$$
\min f(u), \quad g(u) \leq_{K} 0, \quad u \in C .
$$


Let $\bar{u}$ be a local solution of (3.4). "Local" is related to the topology of $L^{\infty}(\Omega)$. To satisfy the regularity condition, we must assume the existence of $u_{0} \in L^{\infty}(\Omega)$ such that (2.4) is fulfilled. We require here a stronger condition and assume that $u_{0}=0$ satisfies (2.4). This means that $\varepsilon>0$ exists such that

$$
\begin{aligned}
& \varepsilon \leq c(x)+\gamma(x) \bar{y}(x)-\gamma(x)\left(G^{\prime}(\bar{u}) \bar{u}\right)(x) \\
& \varepsilon \leq e(x)
\end{aligned}
$$

is satisfied almost everywhere in $\Omega$. This assumption appears to be natural in some sense: the smallest function - namely $u_{0}(x)$ - should satisfy the upper bounds strictly. Since some monotonicity follows from the maximum principle for elliptic equations, this is reasonable. The next result is obtained as a direct conclusion of Theorem 2.4.

LEMMA 3.2. If $\bar{u} \in L^{\infty}(\Omega)$ is a local solution of the optimal control problem (1.1)-(1.3) and the regularity condition (3.5) is satisfied with some $\varepsilon>0$, then $\bar{u}$ is also a solution of the linear optimal control problem

$$
\min f^{\prime}(\bar{u}) u
$$

subject to $u \in L^{\infty}(\Omega)$ and

$$
\begin{aligned}
& u(x) \leq c(x)+\gamma(x)(G(\bar{u}))(x)+\gamma(x)\left(G^{\prime}(\bar{u})(u-\bar{u})\right)(x) \\
& u(x) \leq e(x) \\
& u(x) \geq 0 .
\end{aligned}
$$

Transforming back this problem to an elliptic control problem, we arrive at

$$
\begin{aligned}
\min \{ & \int_{\Omega} \psi_{y}(x, \bar{y}(x), \bar{u}(x)) y(x) d x+\int_{\Gamma} \varphi_{y}(x, \bar{y}(x)) y(x) d s(x) \\
& \left.+\int_{\Omega} \psi_{u}(x, \bar{y}(x), \bar{u}(x)) u(x) d x\right\}
\end{aligned}
$$

subject to the linearized equation (3.1) and

$$
\begin{aligned}
0 \leq u(x) \leq c(x)+\gamma(x) \bar{y}(x)+\gamma(x) y(x)-\gamma(x)\left(G^{\prime}(\bar{u}) \bar{u}\right)(x) \\
u(x) \leq e(x) .
\end{aligned}
$$

We introduce the fixed part of the right-hand side by

$$
\bar{c}(x)=c(x)+\gamma(x) \bar{y}(x)-\gamma(x)\left(G^{\prime}(\bar{u}) \bar{u}\right)(x) .
$$

Moreover, we introduce an adjoint state $\hat{p} \in H^{1}(\Omega) \cap C(\bar{\Omega})$ by

$$
\begin{aligned}
& (A \hat{p})(x)+d_{y}(x, \bar{y}(x)) \hat{p}(x)=\psi_{y}(x, \bar{y}(x), \bar{u}(x)) \quad \text { in } \Omega \\
& \partial_{\nu} \hat{p}(x)+b_{y}(x, \bar{y}(x)) \hat{p}(x)=\varphi_{y}(x, \bar{y}(x)) \quad \text { on } \Gamma \text {. }
\end{aligned}
$$

Then it holds

$$
\int_{\Omega} \psi_{y}(x, \bar{y}(x), \bar{u}(x)) y(x) d x+\int_{\Gamma} \varphi_{y}(x, \bar{y}(x)) y(x) d s(x)=\int_{\Omega} \beta(x) \hat{p}(x) u(x) d x .
$$

This well known relation is easily obtained from the weak formulation of the equations (3.1) and (3.9), respectively. With these notations, the linear programming problem (3.6) becomes equivalent to the primal problem

$$
\min \int_{\Omega} a(x) u(x) d x
$$


subject to

$$
\begin{aligned}
& u(x) \leq \bar{c}(x)+(S u)(x) \\
& u(x) \leq e(x) \\
& u(x) \geq 0
\end{aligned}
$$

with

$$
\begin{aligned}
a & =\beta \hat{p}+\psi_{u}(\cdot, \bar{y}, \bar{u}) \\
S u & =\gamma G^{\prime}(\bar{u}) u .
\end{aligned}
$$

By our assumptions, it holds $a \in L^{\infty}(\Omega)$, and $S$ is a linear continuous operator in $L^{\infty}(\Omega)$. The unknown function is $u \in L^{\infty}(\Omega)$. However, we also know that $\|S u\|_{L^{2}(\Omega)} \leq c\|u\|_{L^{2}(\Omega)}$ so that we can consider $S$ as a linear continuous operator in $L^{2}(\Omega)$. Let us view $S$ in this way and consider the linear programming problem (3.10)-(3.11) for $u \in L^{2}(\Omega)$ with all inequalities defined in $L^{2}(\Omega)$ as well. Notice that this does not change the feasible set, since $0 \leq u \leq e$ implies $u \in L^{\infty}(\Omega)$. Therefore, $\bar{u}$ solves the linear problem (3.11) in $L^{2}(\Omega)$.

4. The dual linear problem. Let us now establish the dual problem associated with the primal problem (3.10)-(3.11). In what follows, we denote by $(\cdot, \cdot)$ the inner product in $L^{2}(\Omega)$, and $S^{*}: L^{2}(\Omega) \rightarrow L^{2}(\Omega)$ denotes the adjoint operator to $S$. By Lagrange duality, the dual problem is obtained as follows: (3.10)-(3.11) is equivalent to

$$
\min _{u \in L^{2}(\Omega)}\left\{\max _{\mu \in\left(L^{2}(\Omega)_{+}\right)^{3}}\left\{(a, u)-\left(u, \mu_{1}\right)+\left(u-S u-\bar{c}, \mu_{2}\right)+\left(u-e, \mu_{3}\right)\right\}\right\},
$$

where $\mu=\left(\mu_{1}, \mu_{2}, \mu_{3}\right)$. The dual problem is obtained by reversing the order of min and $\max$,

$$
\max _{\mu \in\left(L^{2}(\Omega)_{+}\right)^{3}}\left\{\left(-\bar{c}, \mu_{2}\right)-\left(e, \mu_{3}\right)+\min _{u \in L^{2}(\Omega)}\left\{\left(a-\mu_{1}+\mu_{2}-S^{*} \mu_{2}+\mu_{3}, u\right)\right\}\right\} .
$$

The minimum is $-\infty$, if $0 \neq a-\mu_{1}+\mu_{2}-S^{*} \mu_{2}+\mu_{3}$. This is meaningless for the maximization, hence we obtain the dual problem as

$$
\left.\begin{array}{ll} 
& \max \quad-\int_{\Omega}\left(\bar{c}(x) \mu_{2}(x)+e(x) \mu_{3}(x)\right) d x \\
\text { subject to } & \\
& \mu_{2}+\mu_{3}=-a+\mu_{1}+S^{*} \mu_{2} \\
& \mu_{i} \geq 0, \quad i=1,2,3 .
\end{array}\right\}
$$

We show that (4.1) is solvable. Moreover, the problems (3.10)-(3.11) and (4.1) turn out to be in duality - they have the same optimal values. Preparing this discussion, we finish this section by computing $S^{*}$. This operator is defined by $(v, S u)=\left(S^{*} v, u\right)$ $\forall u, v \in L^{2}(\Omega)$. We recall that $S u=\gamma G^{\prime}(\bar{u}) u=\gamma y$, where $y$ is the solution of (3.1). Therefore

$$
(v, S u)=(v, \gamma y)=(\gamma v, y)=(\beta q, u),
$$

where $q$ is the solution of the adjoint equation

$$
\begin{array}{rlll}
(A q)(x)+d_{y}(x, \bar{y}(x)) q(x) & =\gamma(x) v(x) & & \text { in } \Omega \\
\partial_{\nu} q(x)+b_{y}(x, \bar{y}(x)) q(x) & =0 & & \text { on } \Gamma .
\end{array}
$$


This follows again in a standard way from the weak formulation of the equations (3.1), (4.2). Therefore, $S^{*}$ is given by

$$
\left(S^{*} v\right)(x)=\beta(x) q(x),
$$

where $q \in H^{1}(\Omega)$ is the solution of (4.2).

5. The duality relation. Here, we discuss the equality of the optimal values for the primal problem (3.10)-(3.11) and its dual (4.1).

TheOREM 5.1. If $\bar{c}(x) \geq 0$ and $e(x) \geq 0$ hold a.e. in $\Omega$, then the minimum of (3.10)-(3.11) is equal to the supremum of (4.1).

Proof. We sketch the proof for convenience, since it is standard for continuous linear programming problems. We refer, for instance, to [8], [10], [22], [9], to the monography [2] and - in the context of elliptic PDEs - to the recent paper [17].

(i) Define the convex cone $E$,

$$
E=\left\{\left(\alpha, d_{1}, d_{2}\right) \in \mathbb{R} \times L^{2}(\Omega)^{2} \mid \exists u \in L^{2}(\Omega), u \geq 0, u \leq d_{1}+S u, u \leq d_{2},(a, u) \leq \alpha\right\} .
$$

We show that $E$ is closed. In fact, if $\left(\alpha_{n}, d_{1, n}, d_{2, n}\right) \rightarrow\left(\alpha, d_{1}, d_{2}\right)$ in $\mathbb{R} \times L^{2}(\Omega)^{2}$ for $n \rightarrow \infty$, then there are $u_{n} \in L^{2}(\Omega)$ such that

$$
0 \leq u_{n} \leq d_{2, n} \quad \forall n=1,2, \ldots
$$

Therefore, the sequence $\left(u_{n}\right)_{n=1}^{\infty}$ is bounded in $L^{2}(\Omega)$. By weak compactness, we can assume $u_{n} \rightarrow u$ in $L^{2}(\Omega)$. The weak continuity of $S$ yields $S u_{n} \rightarrow S u$ in $L^{2}(\Omega)$. Finally, convexity ensures weak closedness, hence in the limit

$$
0 \leq u \leq d_{2}, \quad u \leq d_{1}+S u, \quad(a, u) \leq \alpha
$$

holds. This means that $\left(\alpha, d_{1}, d_{2}\right)$ is in $E$.

(ii) Let $\bar{\alpha}$ denote the minimum for (3.10)-(3.11). Clearly, the primal problem (3.10)-(3.11) has a solution, i.e. its infimum is attained. This follows from weak compactness, since $0 \leq u \leq e$ gives boundedness of the feasible set. By definition, $\left(\bar{\alpha}-\frac{1}{n}, \bar{c}, e\right) \notin E \forall n \in \mathbb{N}$. Therefore, the element $\left(\bar{\alpha}-\frac{1}{n}, \bar{c}, e\right)$ can be separated from the set $E$ by a closed hyperplane in $\mathbb{R} \times L^{2}(\Omega)^{2}$ : There are $\left(\beta_{n}, c_{n}^{*}, e_{n}^{*}\right) \in \mathbb{R} \times L^{2}(\Omega)^{2}$ such that $\left(\beta_{n}, c_{n}^{*}, e_{n}^{*}\right) \neq(0,0,0)$ and

$$
\beta_{n}\left(\bar{\alpha}-\frac{1}{n}\right)+\left(\bar{c}, c_{n}^{*}\right)+\left(e, e_{n}^{*}\right)<\beta_{n} \alpha+\left(d_{1}, c_{n}^{*}\right)+\left(d_{2}, e_{n}^{*}\right)
$$

holds for all $\left(\alpha, d_{1}, d_{2}\right) \in E$. Inserting $(\bar{\alpha}, \bar{c}, e)$ in the right-hand side, it follows $\beta_{n}>0$ and w.l.o.g. $\beta_{n}=1$. We deduce that $\mu_{2, n}:=c_{n}^{*}$ and $\mu_{3, n}:=e_{n}^{*}$ satisfy the constraints

$$
\begin{aligned}
\mu_{2}+\mu_{3} & \geq-a+S^{*} \mu_{2} \\
\mu_{i} & \geq 0, \quad i=2,3 .
\end{aligned}
$$

To confirm this, insert $d_{1}=u-S u, d_{2}=u$, and $\alpha=(a, u)$ in the right-hand side of (5.1) for arbitrary $u \geq 0$. Denote its (fixed) left-hand side by $\gamma_{n}$. Then

$$
\gamma_{n}<\left(a+\mu_{2, n}+\mu_{3, n}-S^{*} \mu_{2, n}, u\right)
$$

must hold for all $u \geq 0$. This implies $a+\mu_{2, n}+\mu_{3, n}-S^{*} \mu_{2, n} \geq 0$, i.e. the upper inequality of (5.2). Inserting $\alpha=0, u=0, d_{2}=0$, and arbitrary $d_{1} \geq 0$, we obtain

$$
\gamma_{n}<\left(d_{1}, \mu_{2, n}\right) \quad \forall d_{1} \geq 0
$$


and hence $\mu_{2, n} \geq 0$. In the same way, $\mu_{3, n} \geq 0$ is shown.

Setting $\mu_{1}=\mu_{2}+\mu_{3}+a-S^{*} \mu_{2},(5.2)$ becomes equivalent to the constraints of (4.1). Moreover, inserting above $\left(\alpha, d_{1}, d_{2}\right)=(0,0,0) \in E$

$$
-\int_{\Omega}\left(\bar{c}(x) \mu_{2, n}(x)+e(x) \mu_{3, n}(x)\right) d x>\bar{\alpha}-\frac{1}{n}
$$

is obtained for every $n \in \mathbb{N}$. It is true in general that the dual supremum $\bar{\sigma}$ is less or equal the primal minimum $\bar{\alpha}$, cf. (6.13), hence

$$
\bar{\alpha} \geq \bar{\sigma}>\bar{\alpha}-\frac{1}{n} .
$$

Now, $n \rightarrow \infty$ yields $\bar{\alpha}=\bar{\sigma}$, the statement of the theorem.

Remark. The main point in the proof was the closedness of $E$. It was deduced from the boundedness of the set $\left\{u \in L^{2}(\Omega) \mid 0 \leq u \leq e_{n}\right\}$.

6. Solvability of the dual problem. To prove existence for the dual problem, we rely on the nonnegativity and certain smoothing properties of $S$ and $S^{*}$.

Lemma 6.1. $S$ and $S^{*}$ are nonnegative, i.e.

$$
u \geq 0 \Rightarrow S u \geq 0 \text { a.e. in } \Omega \text {. }
$$

This follows from $G^{\prime}(\bar{u}) \geq 0$, a consequence of comparison principles for linear elliptic equations: Testing the linear elliptic equation (3.1) for nonnegative data $u$ by $v^{-}$, the negative part $v^{-}$of $v, v=G^{\prime}(\bar{u}) u$ is seen to vanish. Here, $\beta \geq 0, \gamma \geq 0$ is used.

By their definition, $S$ and $S^{*}$ are linear and continuous operators in $L^{2}(\Omega)$. We shall estimate them in different $L^{p}$-norms for $1 \leq p \leq \infty$. To match their domain $L^{2}(\Omega)$, we have to consider the intersetion of $L^{p}(\Omega)$ with $L^{2}(\Omega)$ in the estimates below. For instance, we know from Theorem 3.1.

$$
\|S u\|_{L^{\infty}(\Omega)} \leq c_{S}\|u\|_{L^{p}(\Omega)} \quad \forall u \in L^{p}(\Omega) \cap L^{2}(\Omega), \quad p \geq N / 2 .
$$

LEMma 6.2. With constants $c_{S}, \delta>0$, the following estimates are fulfilled:

$$
\begin{aligned}
& \left\|S^{*} v\right\|_{L^{\infty}(\Omega)} \leq c_{S}\|v\|_{L^{p}(\Omega)} \quad \forall v \in L^{p}(\Omega) \cap L^{2}(\Omega), \quad p>N / 2 . \\
& \left\|S^{*} v\right\|_{L^{p+\delta}(\Omega)} \leq c_{S}\|v\|_{L^{p}(\Omega)} \quad \forall v \in L^{p}(\Omega) \cap L^{2}(\Omega), \quad 1 \leq p \leq N / 2 .
\end{aligned}
$$

Proof. (i) First estimate: $S^{*} v$ is given by formula (4.3), where $q$ is obtained as the solution of the adjoint elliptic equation (4.2). The mapping $v \mapsto q$ is continuous from $L^{p}(\Omega)$ to $L^{\infty}(\Omega)$. This follows from Theorem 3.1 with $\gamma$ substituted for $\beta$. Therefore, the estimate $\left\|S^{*} v\right\|_{L^{\infty}(\Omega)}=\|\beta q\|_{L^{\infty}(\Omega)} \leq c_{S}\|v\|_{L^{p}(\Omega)}$ follows immediately.

(ii) Some continuity properties: To avoid confusion of operators and their associated spaces, let us first mention that the solution mapping $u \mapsto v$ for equation (3.1) is linear and continuous from $\left(H^{1}(\Omega)^{*}\right)$ to $H^{1}(\Omega)$. Let us call this operator $\mathbf{S}$. In this sense, $S$ is the restriction of $\mathbf{S}$ to $L^{2}(\Omega)$ considered with range in $L^{2}(\Omega)$. Define, for $p>N / 2, S_{p}: L^{p}(\Omega) \rightarrow L^{\infty}(\Omega)$ as the restriction of $\mathbf{S}$ to $L^{p}(\Omega)$. By Theorem 3.1, this operator is continuous. For arbitrary $v \in L^{1}(\Omega)$, the mapping

$$
u \mapsto \int_{\Omega} v\left(S_{p} u\right) d x
$$


defines a functional $\varphi \in L^{p}(\Omega)^{*}=L^{p^{\prime}}(\Omega)$. The mapping $v \mapsto \varphi$ is obviously continuous from $L^{1}(\Omega)$ to $L^{p^{\prime}}(\Omega)$. We denote this mapping by $S_{p}^{\otimes}$. It is the restriction of $S_{p}^{*}$ to $L^{1}(\Omega)$. So we have

$$
\left\|S_{p}^{\otimes} v\right\|_{L^{p^{\prime}}(\Omega)} \leq c_{1}\|v\|_{L^{1}(\Omega)} \quad \forall v \in L^{1}(\Omega)
$$

This estimate holds in particular for all $v \in L^{2}(\Omega)$, and for such functions we have $S_{p}^{\otimes} v=S^{*} v$. In fact, we find for all $u \in L^{\infty}(\Omega)$ and $v \in L^{2}(\Omega)$

$$
\int_{\Omega}\left(S_{p}^{\otimes} v\right) u d x=\int_{\Omega} v\left(S_{p} u\right) d x=\left(v, S_{p} u\right)=(v, S u)=\left(S^{*} v, u\right) .
$$

Since this holds for all $u \in L^{\infty}(\Omega)$, it must hold $S_{p}^{\otimes} v=S^{*} v$ for all $v \in L^{2}(\Omega)$. Therefore, the boundedness of $S_{p}^{\otimes}$ extends to $S^{*}$,

$$
\left\|S^{*} v\right\|_{L^{p^{\prime}}(\Omega)} \leq c_{1}\|v\|_{L^{1}(\Omega)} \quad \forall v \in L^{2}(\Omega) .
$$

This shows that $S^{*}$ can be extended to a continuous linear operator from $L^{1}(\Omega)$ to $L^{p^{\prime}}(\Omega)$. In fact, it is known from Alibert and Raymond [1] or Casas [6] that the solution operator of the adjoint equation (4.2) is linear and continuous from $L^{1}(\Omega)$ to $W^{1, \sigma}(\Omega)$ for all $\sigma<N /(N-1)$. However, we do not rely on this deep result. We just use the information that is available now:

We know in particular that $S^{*}$ is continuous from $L^{p_{0}}(\Omega)$ to $L^{p_{1}}(\Omega)$ and from $L^{q_{0}}(\Omega)$ to $L^{q_{1}}(\Omega)$ for $p_{0}=N, p_{1}=2 N, q_{0}=1, q_{1}=p^{\prime}>1$. It is now fairly clear that the second estimate can be obtained by interpolation.

(iii) Second estimate: Define, for $\theta \in[0,1], r_{j}=r_{j}(\theta)$ by

$$
\frac{1}{r_{j}}=\frac{1-\theta}{p_{j}}+\frac{\theta}{q_{j}}, \quad j=0,1 \text {. }
$$

Then the classical interpolation theorem in Triebel [16], 1.18.7, Thm. 1, ensures that $S^{*}$ is continuous from $L^{r_{0}}(\Omega)$ to $L^{r_{1}}(\Omega)$. From now on, we only need simple calculations. Inserting the concrete values for $p_{j}, q_{j}$, we find

$$
\frac{1}{r_{0}(\theta)}=\frac{1-\theta}{N}+\theta, \quad \frac{1}{r_{1}(\theta)}=\frac{1-\theta}{2 N}+\frac{\theta}{p^{\prime}}
$$

Therefore,

$$
\frac{1}{r_{0}(\theta)}-\frac{1}{r_{1}(\theta)}=\frac{1-\theta}{N}+\theta-\frac{1-\theta}{2 N}-\frac{\theta}{p^{\prime}}=\frac{1}{2 N}+\theta\left(1-\frac{1}{2 N}-\frac{1}{p^{\prime}}\right) .
$$

The difference $1 / r_{0}(\theta)-1 / r_{1}(\theta)$ is a linear function of $\theta$. It admits the values $1 / 2 N$ and $1-1 / p^{\prime}>0$ for $\theta=0$ and $\theta=1$, respectively, thus

$$
\frac{1}{r_{0}(\theta)}-\frac{1}{r_{1}(\theta)} \geq \delta>0
$$

holds with $\delta=\min \left(1 / 2 N, 1-1 / p^{\prime}\right)$. After multiplication with $r_{0} r_{1}>1$ we get $r_{1}(\theta)-r_{0}(\theta) \geq \delta r_{0} r_{1} \geq \delta$ for all $\theta \in[0,1]$, hence $r_{1} \geq r_{0}+\delta$. Therefore, the second estimate holds true for all $r_{0}=p \in[1, N]$, in particular for $p \in[1, N / 2]$. 
TheOREM 6.3. If the regularity condition (3.5) is satisfied, then the dual problem (4.1) has a solution $\bar{\mu} \in\left(L^{\infty}(\Omega)\right)^{3}$.

Proof. (i) Boundedness in $L^{1}$ : We know that the supremum $\bar{\alpha}$ in (4.1) is finite. Let $\left(\mu_{i, n}\right)_{n=1}^{\infty} \in L^{2}(\Omega), i=1,2,3$, be sequences such that the constraints of (4.1) are satisfied and

$$
-\left(\bar{c}, \mu_{2, n}\right)-\left(e, \mu_{3, n}\right) \rightarrow \bar{\alpha}
$$

for $n \rightarrow \infty$. The regularity condition (3.5) gives $\bar{c} \geq \varepsilon, e \geq \varepsilon$ a.e. on $\Omega$, hence

$$
-\left(\bar{c}, \mu_{2, n}\right)-\left(e, \mu_{3, n}\right) \leq-\varepsilon\left(\left\|\mu_{2, n}\right\|_{L^{1}(\Omega)}+\left\|\mu_{3, n}\right\|_{L^{1}(\Omega)}\right) .
$$

Therefore, the $L^{1}$-norm of $\mu_{2, n}$ and $\mu_{3, n}$ is bounded. The boundedness of $\mu_{1, n}$ in $L^{1}(\Omega)$ can now be obtained from the constraints of (4.1).

(ii) Boundedness in $L^{1+\delta}$ : The constraints of (4.1) are equivalent to the constraints (5.2), i.e., $\mu_{2} \geq 0, \mu_{3} \geq 0$, and

$$
\mu_{2}+\mu_{3} \geq-a+S^{*} \mu_{2} .
$$

In view of this, we have

$$
\mu_{2, n}+\mu_{3, n} \geq-a+S^{*} \mu_{2, n}
$$

By Lemma 6.2 and the $L^{1}$-boundedness of the sequence $\mu_{2, n}$, the sequence $\left(S^{*} \mu_{2, n}\right)$ is bounded in the reflexive Banach space $L^{1+\delta}(\Omega)$, hence the right-hand side of $(6.3)$ is bounded in $L^{1+\delta}(\Omega)$. Now, we re-define $\mu_{2, n}, \mu_{3, n}$ as follows: We take

$$
\hat{\mu}_{2, n}(x)+\hat{\mu}_{3, n}(x)= \begin{cases}0 & \text { where }-a(x)+\left(S^{*} \mu_{2, n}\right)(x) \leq 0 \\ -a(x)+\left(S^{*} \mu_{2, n}\right)(x) & \text { where }-a(x)+\left(S^{*} \mu_{2, n}(x)\right)>0\end{cases}
$$

and require $0 \leq \hat{\mu}_{2, n} \leq \mu_{2, n}$ and $0 \leq \hat{\mu}_{3, n} \leq \mu_{3, n}$. Certainly, this is possible. Then,

$$
\left|\hat{\mu}_{2, n}(x)\right|+\left|\hat{\mu}_{3, n}(x)\right| \leq|a|+\left|\left(S^{*} \mu_{2, n}\right)(x)\right| \text {, a.e. on } \Omega,
$$

hence these new sequences are bounded in $L^{1+\delta}(\Omega)$. Moreover, $\hat{\mu}_{2, n}, \hat{\mu}_{3, n} \geq 0$ and $\hat{\mu}_{2, n} \leq \mu_{2, n}$ together with $S^{*} \geq 0$ shows

$$
\hat{\mu}_{2, n}+\hat{\mu}_{3, n} \geq-a+S^{*} \mu_{2, n} \geq-a+S^{*} \hat{\mu}_{2, n},
$$

so that the new functions are feasible. They have a larger or equal objective value than the pair $\mu_{2, n}, \mu_{3, n}$ since $\bar{c} \geq 0$ and $e \geq 0$. In this way, we have found a sequence with better objective value that is bounded in the reflexive Banach space $L^{1+\delta}(\Omega)$. By selecting weakly convergent subsequences we can assume in $L^{1+\delta}(\Omega)$ that

$$
\hat{\mu}_{2, n} \rightarrow \mu_{2}, \quad \hat{\mu}_{3, n} \rightarrow \mu_{3}, \quad n \rightarrow \infty
$$

where $\mu_{2}, \mu_{3} \in L^{1+\delta}(\Omega)$ satisfy all constraints of the dual problem. Moreover, the functions are optimal, and

$$
\mu_{1}:=\mu_{2}+\mu_{3}+a-S^{*} \mu_{2} .
$$

is the associated optimal $\mu_{1}$. 
(iii) Solution in $\left(L^{\infty}(\Omega)\right)^{3}$ : We have found an optimal triplet in $\left(L^{1+\delta}(\Omega)\right)^{3}$ that satisfies the nonnegativity constraints and

$$
\mu_{2}+\mu_{3} \geq-a+S^{*} \mu_{2}
$$

By the smoothing property $(6.2)$ of $S^{*}$, the function $S^{*} \mu_{2}$ belongs to $L^{1+2 \delta}(\Omega)$. Applying the technique of (ii), we find $\hat{\mu}_{2} \geq 0, \hat{\mu}_{3} \geq 0$ which satisfy all constraints. Moreover, they belong to $L^{1+2 \delta}(\Omega)$ and have the same objective value (clearly, the value cannot improved further). Applying this bootstrapping technique, we arrive after finitely many steps to the case where $1+k \delta>N / 2$. In the next step $S^{*}: L^{1+k \delta}(\Omega) \rightarrow L^{\infty}(\Omega)$ is used to have $\hat{\mu}_{i} \in L^{\infty}(\Omega), i=1,2,3$.

THEOREM 6.4. Suppose that $\bar{u}$ is a local solution of the optimal control problem (1.1)-(1.3) that satisfies the regularity condition (3.5). Then there exist Lagrange multipliers $\mu_{i} \in L^{\infty}(\Omega), i=1,2,3$, satisfying with $\bar{u}, \bar{y}, \bar{p}$ the following optimality system that consists of the constraints (1.2)-(1.3), the adjoint equation

$$
\begin{aligned}
(A p)(x)+d_{y}(x, \bar{y}(x)) p(x) & =\psi_{y}(x, \bar{y}(x), \bar{u}(x))-\gamma(x) \mu_{2}(x) & & \text { in } \Omega \\
\partial_{\nu} p(x)+b_{y}(x, \bar{y}(x)) p(x) & =\varphi_{y}(x, \bar{y}(x)) & & \text { on } \Gamma
\end{aligned}
$$

and, for almost all $x \in \Omega$, the conditions

$$
\begin{aligned}
\psi_{u}(x, \bar{y}(x), \bar{u}(x))+\beta(x) p(x)+\mu_{3}(x)+\mu_{2}(x)-\mu_{1}(x) & =0 \\
\mu_{i}(x) & \geq 0 \\
(\bar{u}(x)-e(x)) \mu_{3}(x) & =0 \\
(\bar{u}(x)-\gamma(x) \bar{y}(x)-c(x)) \mu_{2}(x) & =0 \\
\bar{u}(x) \mu_{1}(x) & =0 .
\end{aligned}
$$

Proof. We consider the optimal solutions $\mu_{i} \in L^{\infty}(\Omega)$ of the dual problem (4.1) and show that they fulfill the required properties. First, we mention

$$
\begin{aligned}
(a, \bar{u}) & \geq(a, \bar{u})+\left(\bar{u}-\bar{c}-S \bar{u}, \mu_{2}\right)+\left(\bar{u}-e, \mu_{3}\right) \\
& =\left(a+\mu_{2}+\mu_{3}-S^{*} \mu_{2}, \bar{u}\right)-\left(\bar{c}, \mu_{2}\right)-\left(e, \mu_{3}\right) \\
& \geq-\left(\bar{c}, \mu_{2}\right)-\left(e, \mu_{3}\right),
\end{aligned}
$$

since $\bar{u}, \mu_{2}, \mu_{3} \geq 0$ and the inequalities (3.11), (6.5) hold true. From the duality relation proved in Theorem 5.1,

$$
(a, \bar{u})=-\left(\bar{c}, \mu_{2}\right)-\left(e, \mu_{3}\right),
$$

we deduce that the inequalities (6.12), (6.13) must hold as equations, hence

$$
0=\left(\bar{u}-\bar{c}-S \bar{u}, \mu_{2}\right)=\left(\bar{u}-e, \mu_{3}\right)=\left(a+\mu_{2}+\mu_{3}-S^{*} \mu_{2}, \bar{u}\right) .
$$

By definition, $\bar{c}=c+\gamma \bar{y}-S \bar{u}$, hence (6.14) yields

$$
\int_{\Omega}(\bar{u}-c-\gamma \bar{y}) \mu_{2} d x=\int_{\Omega}(\bar{u}-e) \mu_{3} d x=0 .
$$

Thanks to $\bar{u}-c-\gamma \bar{y} \leq 0, \bar{u} \leq e$ and the nonnegativity of $\mu_{2}, \mu_{3}$ this is equivalent to (6.9)-(6.10). Inserting $\mu_{1}=a+\mu_{2}+\mu_{3}-S^{*} \mu_{2}$, we obtain from (6.14)

$$
\int_{\Omega} \bar{u} \mu_{1} d x=0
$$


The nonnegativity of $\bar{u}$ and $\mu_{1}$ implies (6.11). By definition, it holds

$$
a+\mu_{2}+\mu_{3}-\mu_{1}-S^{*} \mu_{2}=0 .
$$

The representation (4.3) for $S^{*}$ permits to write

$$
S^{*} \mu_{2}=\beta q,
$$

where $q$ solves the adjoint equation (4.2) with right-hand side $\gamma \mu_{2}$. Moreover

$$
a=\psi_{u}(\bar{y}, \bar{u})+\beta \hat{p},
$$

where $\hat{p}$ is the solution of (3.9). Obviously, (6.15)-(6.17) are equivalent to (6.6)-(6.7). The complete system of necessary conditions is shown.

7. An application to pointwise state constraints. Consider the optimal control problem (1.1)-(1.2) subject to the pointwise constraints

$$
\begin{aligned}
& 0 \leq u(x) \\
& 0 \leq c(x)+y(x) .
\end{aligned}
$$

We know $y \in C(\bar{\Omega})$, hence a Lagrange multiplier $\mu_{2}$ associated with (7.2) is in general a regular Borel measure provided that $c \in C(\bar{\Omega})$, cf. Casas [6]. One way to deal with (7.2) numerically, is the Lavrentiev type regularization

$$
-\lambda u(x) \leq c(x)+y(x)
$$

with $\lambda>0$ being small [11].

For the results of this section, we assume that the control problem (1.1), (1.2), (7.1), (7.3) has a locally optimal solution $\bar{u} \in L^{\infty}(\Omega)$. Notice that this boundedness does not follow from the constraints (7.1), (7.3).

We do not consider here the pass to the limit $\lambda \downarrow 0$. However, we are able to show that, for $\lambda>0$ fixed, the associated Lagrange multiplier $\mu_{2}$ can be taken as a function from $L^{\infty}(\Omega)$ again. For this result, the theory of the preceding sections needs a few modifications. To simplify the notation, we write $\mu:=\mu_{2}$ below. Recall that now $\gamma(x)=1, S=G^{\prime}(\bar{u})$ and therefore $\bar{c}=c+\bar{y}-S \bar{u}$.

The state constraints in (7.2) are only meaningful, if $c \geq 0$, since otherwise $u \geq 0$ would imply $c+y \geq 0$. Therefore, we cannot assume $\bar{c} \geq 0$ now, if we are interested in taking $\lambda$ small (consider, for instance, the linear case, where $\bar{y}=S \bar{u}$ and hence $c=\bar{c})$.

Our approach is based on linearization and needs the regularity condition (2.4). For the case of the constraints (7.1),(7.3), this condition amounts to the existence of $u_{0} \in L^{\infty}(\Omega)$ and $\varepsilon>0$ such that

$$
\varepsilon \leq u_{0}(x), \quad \varepsilon-\lambda u_{0}(x) \leq \bar{c}(x)+S u_{0}(x)
$$

for almost all $x \in \Omega$. It is easy to see that this condition is satisfied for the function $u_{0}(x) \equiv c_{0}$, if $c_{0}$ is taken sufficiently large. Indeed, for $c_{0} \rightarrow \infty$, the term $-\lambda c_{0}$ in (7.4) tends to $-\infty$, while $S c_{0}$ remains nonnegative. Moreover, $\bar{c}$ is bounded. Therefore, (7.4) is satisfied for sufficiently large $c_{0}$, and we are justified to linearize at any $\bar{u}$.

The associated linear programming problem for a local solution of (1.1)-(1.2), (7.2), (7.3) which replaces (3.10)-(3.11), is now

$$
\begin{aligned}
\min & (a, u) \\
-\lambda u \leq \bar{c}+S u & \\
u \geq & 0, u \in L^{\infty}(\Omega) .
\end{aligned}
$$


We know that $\bar{u}$ is a solution of that problem, since $\bar{u}$ is supposed to be a local solution of the optimal control problem in $L^{\infty}(\Omega)$ and the regularity condition is satisfied. However, this information is related to the space $L^{\infty}(\Omega)$ that is not suitable to obtain regular Lagrange multipliers. Therefore, we show that the optimal value of the problem (7.5) does not increase, if the feasible set is extended from $L^{\infty}(\Omega)$ to $L^{p}(\Omega)$ with $p>N / 2$.

LEMMA 7.1. The optimal value of problem (7.5) does not change, if its feasible set is extended to all $u \in L^{p}(\Omega), p>\max \{2, N / 2\}$, satisfying the associated constraints.

Proof. Let an arbitrary $u \in L^{p}(\Omega)$ be given such that $u \geq 0$ and

$$
-\lambda u \leq \bar{c}+S u \quad \text { a.e. in } \Omega
$$

We construct a sequence of functions $u_{n} \in L^{\infty}(\Omega)$ such that $u_{n}$ satisfies (7.6) and $u_{n} \rightarrow u$ in $L^{p}(\Omega)$ as $n \rightarrow \infty$. This implies the statement of the Lemma.

By the smoothing property (6.2) of $S$, we know $\bar{c}+S u \in C(\bar{\Omega})$. Define

$$
K=\frac{1}{\lambda}\|\bar{c}+S u\|_{L^{\infty}(\Omega)}
$$

and introduce the "cut-off" function

$$
v_{n}(x)=\left\{\begin{aligned}
n, & u(x)>n \\
u(x), & u(x) \leq n
\end{aligned}\right.
$$

This function is bounded, measurable and nonnegative. Then $v_{n} \rightarrow u$ holds in $L^{p}(\Omega)$ as $n \rightarrow \infty$. For all $n \geq K$ we have

$$
-\lambda v_{n} \leq \bar{c}+S u \quad \text { a.e. in } \Omega \text {. }
$$

This inequality is trivial for all $x$ with $u(x) \leq n$, since $v_{n}(x)=u(x)$ holds there. In the remaining points, it holds

$$
-\lambda v_{n}(x)=-\lambda n \leq-\lambda K=-\|\bar{c}+S u\|_{L^{\infty}(\Omega)} \leq \bar{c}(x)+(S u)(x) .
$$

In view of $v_{n} \rightarrow u$, the continuity of $S$ from $L^{p}(\Omega)$ to $C(\bar{\Omega})$ yields

$$
-\lambda v_{n} \leq \bar{c}+S v_{n}+\varepsilon_{n}
$$

with $\varepsilon_{n} \downarrow 0$ as $n \rightarrow \infty$. For sufficiently large constant $c_{0}>0$, we have

$$
-\lambda c_{0} \leq \bar{c}+S c_{0}-1
$$

For all $t \in[0,1]$, the convex combination $u_{n}:=(1-t) v_{n}+t c_{0}$ is nonnegative and satisfies

$$
-\lambda u_{n} \leq \bar{c}+S u_{n}+(1-t) \varepsilon_{n}-t
$$

We take $t=t_{n}=\varepsilon_{n} /\left(1+\varepsilon_{n}\right)$. Then $\left(1-t_{n}\right) \varepsilon_{n}-t_{n}=\varepsilon_{n}-t_{n}\left(1+\varepsilon_{n}\right)=0$, thus

$$
-\lambda u_{n} \leq \bar{c}+S u_{n}
$$

hence $u_{n}$ satisfies the linearized constraints. Moreover, it holds $u_{n} \rightarrow u, n \rightarrow \infty$. 
Lemma 7.1 ensures that $\bar{u}$ is also a solution of the extended primal problem

$$
\begin{aligned}
& \min (a, u) \\
&-\lambda u \leq \bar{c}+S u \\
& u \geq 0, u \in L^{p}(\Omega) .
\end{aligned}
$$

The dual problem to $(7.7)$ is

$$
\begin{aligned}
& \max (-\bar{c}, \mu) \\
& a \geq \lambda \mu+S^{*} \mu \\
& \mu \geq 0, \mu \in L^{p^{\prime}}(\Omega),
\end{aligned}
$$

where a function $\mu \in L^{p^{\prime}}(\Omega)$ is to be found. We have already discussed that $S_{p}^{\otimes}$ maps $L^{1}(\Omega)$ to $L^{p^{\prime}}(\Omega)$, hence $S^{*}$ can be extended to a continuous operator in $L^{p^{\prime}}(\Omega)$. Moreover, $S^{*}$ is nonnegative. We shall see that the constraints above imply $\mu \in$ $L^{\infty}(\Omega)$, hence $\mu \in L^{2}(\Omega)$ so that the notation $S^{*}$ in $(7.8)$ is justified.

We show that both problems admit the same optimal values and that (7.8) has a solution.

LEMMA 7.2. The function $a$ is nonnegative a.e. on $\Omega$.

Proof. As the regularity condition is always satisfied here, Theorem 2.4 shows that $\bar{u}$ solves the linearized problem (7.5). Moreover, Lemma 7.1 ensures $\bar{u}$ to be a solution of the extended problem (7.7), too. Therefore, (7.7) must have at least the optimal solution $\bar{u}$. For all $u \geq 0, \bar{u}+u$ satisfies the constraints of (7.5): Obviously, we have $\bar{u}+u \geq 0$. Moreover,

$$
-\bar{c} \leq \lambda \bar{u}+S \bar{u} \leq \lambda(\bar{u}+u)+S(\bar{u}+u)
$$

follows from $\lambda \geq 0$ and $S \geq 0$. Assume that $a(x)<0$ on $M \subset \Omega$, where $M$ has positive measure. Take

$$
u(x)= \begin{cases}1 & \text { on } M \\ 0 & \text { on } \Omega \backslash M .\end{cases}
$$

Then $\bar{u}+u$ is feasible, but $(a, \bar{u}+u)<(a, \bar{u})$ holds in contrary to the optimality of $\bar{u}$.

TheOREM 7.3. The dual problem (7.8) admits a solution. Moreover, the maximum of the primal problem (7.5) is equal to the minimum of (7.8). The solution of (7.8) is bounded and measurable and is a Lagrange multiplier associated with (7.2).

Proof. (i) Solvability of (7.8): We know from Lemma 7.2 that $a$ is nonnegative. Therefore, $\mu=0$ is feasible for (7.8). The feasible set is bounded in $L^{\infty}(\Omega)$, since $\mu \geq 0$ implies $S^{*} \mu \geq 0$ and hence

$$
a \geq \lambda \mu+S^{*} \mu \geq \lambda \mu \geq 0 .
$$

Now the existence of an optimal solution of (7.8) follows by weak*-compactness.

(ii) Equality of optimal values: Re-write (7.8) in the form of a primal problem, namely

$$
\begin{aligned}
\min & \left(\bar{c}, \mu_{2}\right) \\
\lambda \mu+S^{*} \mu & \leq a \\
\mu \geq 0 . &
\end{aligned}
$$


Its dual has the form

$$
\begin{aligned}
\max & (-a, u) \\
\lambda u+S u & \geq-\bar{c} \\
u & \geq 0 .
\end{aligned}
$$

(If $B$ is a linear continuous operator, then the general rule for establishing dual problems is as follows: If the primal problem is to minimize $(c, u)$ subject to $B u \leq a$ and $u \geq 0$, then is dual is to maximize $(-a, \mu)$ subject to $B^{*} \mu \geq-c$ and $\mu \geq 0$.)

The optimal values of (7.9) and (7.10) are equal. To show this, we follow the same steps as in the proof of Theorem 5.1 We introduce the cone

$$
E:=\left\{(\alpha, d) \in \mathbb{R} \times L^{p^{\prime}}(\Omega) \mid \exists \mu \geq 0: \lambda \mu+S^{*} \mu \leq d,(\bar{c}, \mu) \leq \alpha\right\} .
$$

Again, $E$ is closed (boundedness of a sequence $\left\{\alpha_{n}, d_{n}\right\}$ in $\mathbb{R} \times L^{p^{\prime}}(\Omega)$ implies that the associated sequence $\left\{\mu_{n}\right\}$ is bounded). Moreover, the element $\left(\bar{\alpha}-\frac{1}{k}, \bar{c}\right)$ does not belong to $E$ for all $k \in \mathbb{N}$. This element can be separated from $E$ by a closed hyperplane. Continuing as in the proof of Theorem 5.1 (here, the term $e$ does not occur) the equality of the optimal values of (7.9) and (7.10) is obtained. By changing the signs, the same holds for the problems (7.8) and (7.7). Lemma 7.1 completes the proof. It follows as in Section 6 that the solution $\mu \in L^{\infty}(\Omega)$ of $(7.8)$ is a Lagrange multiplier associated with the mixed pointwise control-state constraint (7.2).

\section{REFERENCES}

[1] J.-J. Alibert AND J.-P. RAymond, Boundary control of semilinear elliptic equations with discontinuous leading coefficients and unbounded controls, Numer. Funct. Anal. and Optimization, 3\&4 (1997), pp. 235-250.

[2] E. J. Anderson, Linear programming in infinite-dimensional spaces. Theory and applications., Wiley, Chichester, 1987.

[3] N. Arada And J. P. Raymond, Optimal control problems with mixed control-state constraints, SIAM J. Control, 39 (2000), pp. 1391-1407.

[4] M. Bergounioux And F. Tröltzsch, Optimal control of linear bottleneck problems, ESAIM, Control, Optimisation and Calculus of Variations, 3 (1998), pp. 235-250.

[5] - Optimal control of semilinear parabolic equations with state-constraints of bottleneck type, ESAIM, Control, Optimisation and Calculus of Variations, 4 (1999), pp. 595-608.

[6] E. CASAS, Boundary control of semilinear elliptic equations with pointwise state constraints, SIAM J. Control and Optimization, 31 (1993), pp. 993-1006.

[7] E. Casas, F. Tröltzsch, AND A. Unger, Second order sufficient optimality conditions for some state-constrained control problems of semilinear elliptic equations, SIAM J. Control and Optimization, 38 (2000), pp. 1369-1391.

[8] R. Grinold, Continuous programming I, J. Math. Analysis Appl., 28 (1969), pp. 39-51.

[9] W. KRABS, Lineare Optimierung in halbgeordneten Vektorräumen, Numerische Mathematik, 11 (1968), pp. 220-231.

[10] N. Levinson, A class of continuous linear programming problems, J. Math. Analysis Appl., 16 (1966), pp. 73-83.

[11] C. Meyer, A. Rösch, And F. Tröltzsch, Optimal control of PDEs with regularized pointwise state constraints, tech. rep., Inst. of Math., Technische Universität Berlin, 2003. Report 14-2003, to appear in Computational Optimization and Applications.

[12] M. K. V. Murthy and G. Stampacchia, A variational inequality with mixed boundary conditions, Israel J. Math., 13 (1972), pp. 188-224.

[13] J.-P. PENOt, On regularity conditions in mathematical programming, Mathematical Programming Study, 19 (1982), pp. 167-199.

[14] A. Rösch AND F. TRÖLTzsCH, Sufficient second order optimality condititions for an elliptic optimal control problem with pointwise control-state constraints, 2004, submitted.

[15] — Sufficient second order optimality conditions for a parabolic optimal control problem with pointwise control-state constraints, SIAM J. Control and Optimization, 42 (2003), pp. 138-154. 
[16] H. Triebel, Interpolation Theory, Function Spaces, Differential Operators, J. A. Barth Verlag, Heidelberg-Leipzig, 1995.

[17] F. TRÖLtzSCH, Regular Lagrange multipliers for control problems with mixed pointwise controlstate constraints, SIAM J. on Optimization, to appear.

[18] — A minimum principle and a generalized bang-bang-principle for a distributed optimal control problem with constraints on the control and the state, ZAMM, 59 (1979), pp. 737739 .

[19] - The generalized bang-bang-principle and the numerical solution of a parabolic boundarycontrol problem with constraints on the control and the state, ZAMM, 64 (1984), pp. 551557.

[20] - Optimality conditions for parabolic control problems and applications, vol. 62 of Teubner Texte zur Mathematik, Teubner-Verlag, Leipzig, 1984.

[21] _ Optimale Steuerung partieller Differentialgleichungen-Theorie, Verfahren und Anwendungen, Vieweg, Wiesbaden, 2005.

[22] W. TyNDALL, A duality theorem for a class of continuous linear programming problems, J. SIAM, 13 (1965), pp. 644-666.

[23] J. Zowe AND S. KurCYUsz, Regularity and stability for the mathematical programming problem in Banach spaces, Appl. Math. Optimization, 5 (1979), pp. 49-62. 